ROBERT BARBAULT

\section{INTRODUCTION}

Confronté à la multitude des espèces qui composent tout écosystème le gestionnaire, comme le chercheur, est tenté de s'y repérer en fixant son attention sur un petit nombre d'entre elles, les plus importantes. En fait, le choix est généralement biaisé et exprime les intérêts ou les goûts particuliers de l'observateur. Au mieux, il résulte d'une sélection effectuée en fonction de la question posée. Tel forestier privilégiera par exemple les essences d'arbre à valeur économique ; tel entomologiste une série d'espèces caractéristiques du site, carabes ou papillons ; tel botaniste enfin, des espèces menacées d'extinction. D'une manière générale les projets de restauration sont exposés aux mêmes risques de subjectivité, inconsciente ou non. I1 y a là une source d'échecs évidente, pour des raisons écologiques aussi bien que pour des raisons socio-économiques.

Restaurer implique en effet l'idée d'une situation, d'un état, que l'on veut recouvrir. I1 y a une référence à un passé qui, s'inscrivant nécessairement dans l'histoire d'une région, relève de la société plus que de la nature. La démarche à engager est double :

\title{
RÉSUMÉ : Le concept d'espèce-clé de voûte en écologie de la restauration : clé... ou impasse ?
}

Que I'on cherche à en comprendre le fonctionnement ou que l'on se propose de les gérer ou de les restaurer, les systèmes écologiques nous opposent, par la multitude des espèces qui les composent, une difficulté majeure. Espérer surmonter l'obstacle en réduisant de tels ensembles hyperdiversifiés à quelques niveaux trophiques apparaît aussi peu satisfaisant que l'accumulation indéfinie de monographies d'espèces.

Né dans les années soixante, le concept d'espèce clé de voûte connaît aujourd'hui un nouvel engouement : dans la mouvance des débats actuels sur la conservation de la biodiversité il apparaît comme une voie d'accès privilégiée à la complexité évoquée ci-dessus. Parce qu'il postule que certaines espèces sont plus importantes que d'autres, jouant un rôle pivot ou carrefour dans les réseaux trophiques, ce concept laisse penser que l'on pourrait ainsi orienter l'attention et les efforts sur un petit nombre d'espèces structurantes, négligeant toutes les autres qui en dépendent directement ou indirectement.

En fait, il semble bien que ce concept soulève plus de difficultés qu'il n'en résout. Si il ne constitue pas par lui-même un outil miracle directement utilisable en écologie de la restauration, il y apparaît néanmoins comme structurant par les idées sous-jacentes qu'il véhicule:

- l'idée de système écologique (qui s'oppose à la perception première d'une multitude d'espèces incoordonnées) ;

- l'idée d'interactions entre espèces (conséquence logique du premier point) ;

- le concept de système population-environnement qui intègre tous les acquis de la biologie des populations.

Aussi, sans constituer une clé miraculeuse mais loin d'être une impasse, le concept d'espèce clé de voûte conduit finalement à d'intéressantes perspectives pour l'écologie de la restauration.
Résumé en anglais p. 28

- Il faut définir (ou s'entendre sur) l'objectif que l'on se donne (quel état restaurer? Quel est le passé que l'on prend comme référence pour un "retour aux origines" ?).

- Il faut élaborer une trajectoire devant conduire de l'état actuel, considéré comme dégradé, à l'état de référence. Tout cela suppose une vision dynamique et une approche systémique appliquées à des ensembles écologiques, ce qui correspond bien aux compétences et à la pratique actuelle de cette science ; mais la perspective ne peut être donnée que par les sciences de l'homme et de la société : le cadre, les valeurs, le référentiel sont d'ordre social - ou déterminés par le contexte socio-économique.

Quoi qu'il en soit, que l'on cherche à en comprendre le fonctionnement ou que l'on se propose de les gérer ou de les res. taurer, les systèmes écologiques nous opposent, par la multitude des espèces qui les composent, une difficulté majeure. Espérer surmonter l'obstacle en réduisant de tels ensembles hyperdiversifiés à quelques niveaux trophiques' apparait aussi peu satisfaisant que l'accumulation indéfinie de monographies d'espèces.

Est-il possible de développer une approche objective permettant d'établir une hiérarchie entre toutes les espèces qui composent un écosystème? Peut-on distinguer a priori et objectivement des espèces plus importantes que d'autres? C'est ce que prétend le concept d'espèce-clé de voûte. Est désignée ainsi toute espèce dont la présence a des effets majeurs sur la persistance 
d'autres espèces. Le critère d'importance, implicite ici, est d'ordre écologique : c'est le maintien du système considéré, avec sa biodiversité maximale ou au moins avec un fonctionnement durable.

En fait, il semble bien que ce concept soulève plus de difficultés qu'il n'en résout. De pragmatique qu'il était au départ, il souffre d'être devenu trop vite - presque sous l'effet d'urgence - théorique (voir Bond, 1993 ; Mills et al., 1993). Ainsi pour Mills, Soulé et Doak (1993), la complexité des interactions écologiques et la méconnaissance que l'on en a ne militent pas en faveur d'une utilisation pratique de ce concept en matière d'aménagement ou de restauration. Mais si il ne constitue donc pas par luimême un outil miracle directement utilisable en écologie de la restauration, il y apparaît néanmoins comme structurant par les idées sous-jacentes qu'il véhicule. N'oublions pas, en effet, qu'il n'est d'abord qu'un sous-produit particulier d'une repré.

sentation construite des systèmes écologiques, je veux parler des réseaux trophiques (encadré). Les trois idées dont on peut ici tirer parti sont :

- l'idée de système écologique (qui s'oppose à la perception première d'une multitude d'espèces incoordonnées) ;

- l'idée d'interactions entre espèces (conséquence logique du premier point) ;

- le concept de système population. environnement, qui intègre tous les acquis de la biologie des populations.

Aussi, sans constituer une clé miraculeuse, le concept d'espèce clé de voûte est loin d'être une impasse : il offre indirectement d'intéressantes perspectives pour l'écologie de la restauration... une discipline encore à construire !

\section{DU CONCEPT À UNE TYPOLOGIE DES ESPĖCES-CLÉ DE VOÛTE}

Né dans les années soixante, le concept d'espèce-clé connaît aujourd'hui un nouvel engouement : dans la mouvance des débats actuels sur la conservation de la biodiversité il brille comme un soleil d'espoir

\section{POPULATION, SYSTÈME POPULATION/ENVIRONNEMENT ET RÉSEAU TROPHIQUE}

La population, ensemble des individus de même espèce occupant un même écosystème, est l'unité élémentaire fondamentale des systèmes populations interconnectées.

Cependant, si les populations naturelles sont bien les pièces élémentaires à partir desquelles se structurent les écosystèmes, elles ne constituent pas, à elles seules, des unités fonctionnelles, étudiables isolément. Ainsi, du point de vue du fonctionnement et de l'évolution, le plus petit objet que puisse considérer un écologue est ce que l'on conviendra d'appeler un système population-environnement.

Considérons le schéma de la figure 1 qui représente un réseau trophique simple, noyau l'intérieur de l'écosystème auquel elle appartient, toute population peut présenter avec son environnement cinq types principaux de relations. D'une part elle participe à des interactions écologiques: les écosystèmes sont composés de structurel type des systèmes écologiques. A verticales avec ses proies et ses prédateurs ou parasites et à des interactions horizontales avec d'autres populations appartenant au même niveau trophique qu'elle (relations de compétition). D'autre part elle est soumise directement et indirectement aux effets des facteurs physiques et chimiques du milieu tandis qu'elle peut profiter d'interactions positives (mutualisme ou symbiose) avec d'autres espèces. Enfin, des processus intrinsèques peuvent aussi jouer population-environnement et réseaux trophiques sont des représentations construites, c'est-à-dire des interprétations des assemblages écologiques que l'on observe à la surface de la planète, sur la terre ou dans les mers. Les premiers résultent de préoccupations espèces(s)centrées tandis que les seconds prétendent traduire la totalité des interactions qui relient l'ensemble des espèces présentes à un moment donné, dans un espace déterminé. un rôle dans sa dynamique. Ainsi, systèmes

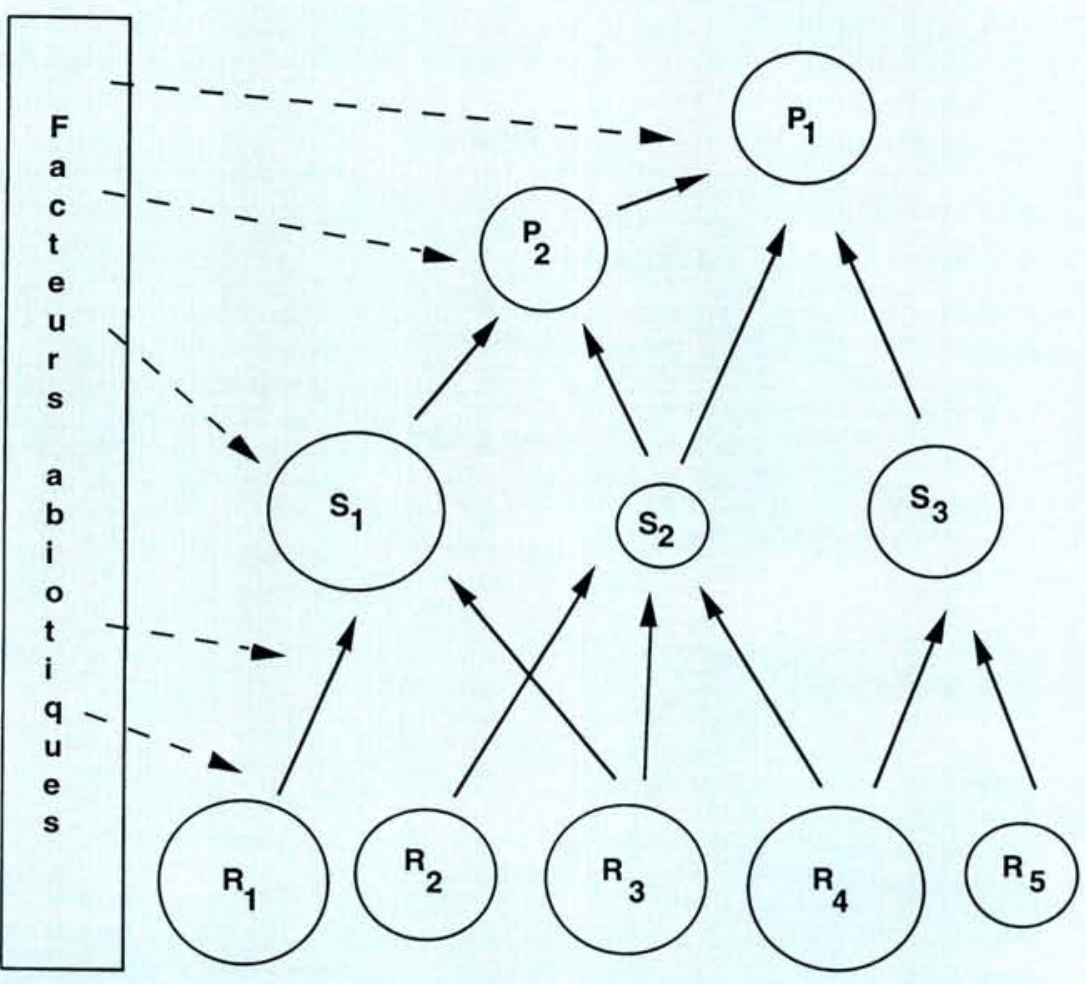

Figure 1 - Représentation simplifiée d'un système écologique centré sur un réseau trophique composé de trois espèces $\mathbf{S 1}, \mathbf{S 2}$ S3 se partageant des ressources Ri et soumis à deux espèces prédatrices P1 et P2. 
dans la jungle obscure évoquée ci-dessus. Le concept d'espèce-clé de voûte est attribué à Paine $(1966,1969)$. Pour cet auteur les espèces clé de voûte sont des espèces dont l'activité et l'abondance déterminent "the integrity of the community and its unaltered persistence through time, that is, stability". Le retrait expérimental d'une espèce-clé de voûte, comme celui de l'étoile de mer Pisaster ochraceus effectué par Robert Paine dans une communauté intertidale de la côte Pacifique des États-Unis, doit se traduire par la disparition d'autres espèces ( 7 sur 15 dans l'exemple cité) et leur remplacement éventuel par quelques autres (ici, l'extension de la moule de Californie). Parce qu'il postule que certaines espèces sont plus importantes que d'autres, jouant un rôle pivot ou carrefour dans les réseaux trophiques, ce concept laisse penser que l'on pourrait ainsi orienter l'attention et les efforts sur un petit nombre d'espèces structurantes, négligeant toutes les autres qui en dépendent directement ou indirectement (encadré).

Dans le cadre des préoccupations relatives à la conservation de la biodiversité (voir Barbault, 1994) le concept d'espèce-clé connait aujourd'hui un net regain. On en trouve une revue générale orthodoxe dans
Bond (1993) et une approche plus critique sous la plume de Mills et al. (1993).

Le tableau 1 propose une classification schématique des divers types d'espèces clé de voûte.

D'abord clair avec la démonstration expérimentale qu'en a donnée Robert Paine à propos de réseaux trophiques marins, le concept d'espèce-clé s'efface peu à peu à mesure que l'on tente de le généraliser. La mise en évidence du rôle structurant de prédateurs de sommet, qu'il s'agisse d'étoiles de mer comme dans le cas précédent ou des rats kangourous granivores mis en vedette dans les travaux expérimentaux rapportés par Brown et Heske (1990), a marqué un tournant dans l'écologie des communautés, un peu écrasée par le conformisme dominant des interprétations fondées sur des arguments de type "compétition", "partage des ressources"; on redécouvrait les prédateurs, en attendant de le faire pour les parasites et pathogènes qui allait demander une décennie de plus pour s'imposer (Barbault, 1992).

Ainsi, les prédateurs peuvent exercer un rôle clé dans la structuration de communautés naturelles, notamment en limitant la prolifération de certaines espèces compétitivement dominantes et en favorisant

\section{LE CONCEPT D'ESPÈCE-CLÉ DE VOUTE DE R.T. PAINE}

Le terme de keystone species a été introduit par R.T. Paine en 1969, mais le phénomène avait été clairement exposé dans un article antérieur (1966) abondamment cité depuis.

Sur les bancs rocheux de la zone intertidale des côtes américaines Paine relève l'association remarquablement constante de moules, de balanes et d'une étoile de mer faisant fonction de prédateur de sommet, du moins à cette échelle d'analyse de la communauté intertidale d'invertébrés. La figure 2 souligne les relations trophiques qui caractérisent cette fraction de la communauté telle qu'elle est observée à Washington.

En juin 1963, R.T. Paine élimine l'étoile de mer du banc rocheux sur des bandes de $8 m \times 2 m$. Dès septembre il observe l'expansion de Balanus glandula, qui occupe selon les sites 60 à $80 \%$ de l'espace disponible. En juin de l'année suivante les balanes étaient repoussés par la croissance rapide de la moule Mytilus californianus qui domina peu à peu tout l'espace avec la subsistance sporadique de Mitella (balane).

Ainsi, la présence du prédateur de sommet permettait la coexistence de nombreuses espèces d'invertébrés en compétition pour la colonisation de la bande rocheuse située dans la zone de balancement des marées. Sa disparition entraîne toujours un appauvrissement de la communauté intertidale par exclusion compétitive des espèces les moins efficaces à coloniser le substrat rocheux. L'étoile de mer Pisaster ochraceus est une espèce clé de voûte et l'hypothèse de Paine est, selon ses propres termes, que "local species diversity is directly related to the efficiency with which predators prevent the monopolization of the major environmental requisites by one species".
1. Il est classique en effet, pour représenter les écosystèmes, de regrouper l'ensemble des espèces en quelques compartiments fonctionnels définis sur la base des flux de matière et d'énergie. On définit ainsi l'ètage des producteurs primaires (qui regroupe toutes les plantes vertes, sans distinction d'especes) puis, celui des consommateurs primaires (les herbivores), des consommateurs secondaires (les carnivores qui se nourrissent d'herbivores), etc. 

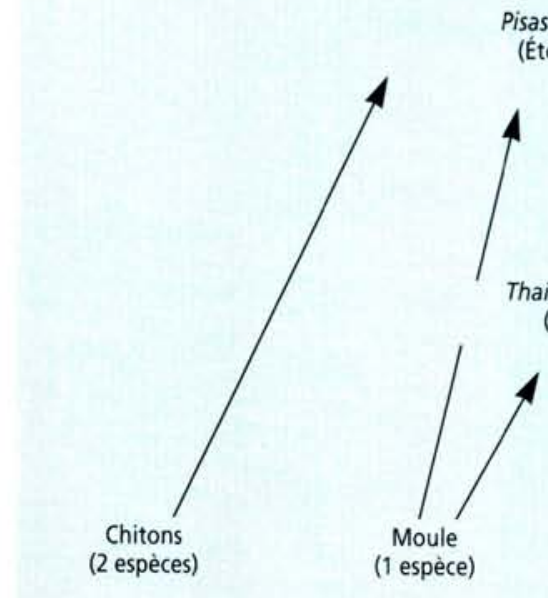
er ochraceus (Étoile de mer)

Figure 2-Représentation partielle du réseau trophique dominé par l'étoile de mer Pisaster ochraceus à Mukkaw Bay (d'après Paine, 1966).

Toutes les espèces ne sont pas représentées. Les crustacés ou mollusques qui constituent ici l'étage inférieur sont des brouteurs d'algues ou des filtreurs (comme la moule).

\begin{tabular}{|c|c|c|}
\hline Type & Mode d'action & Exemples \\
\hline $\begin{array}{l}\text { Prédateurs (carnivores } \\
\text { ou herbivores). }\end{array}$ & $\begin{array}{l}\text { Favorisent la coexistence d'espèces } \\
\text { potentiellement compétitives. }\end{array}$ & $\begin{array}{l}\text { Étoiles de mer (Paine, 1966, 1980). } \\
\text { Loutre de mer et oursins (Estes et al., 1978). } \\
\text { Rongeurs granivores (Brown et Heskes, 1990) }\end{array}$ \\
\hline Proies. & $\begin{array}{l}\text { Permettent le développement de } \\
\text { prédateurs ou d'herbivores et la survie } \\
\text { d'autres espèces que, du fait de leur } \\
\text { présence, ceux-ci ne surconsomment pas. }\end{array}$ & Nombreuses plantes (Terborgh, 1986). \\
\hline Mutualistes. & $\begin{array}{l}\text { Favorisent le maintien des espèces } \\
\text { auxquelles ils sont associés - et de } \\
\text { toutes celles qui en dépendent. }\end{array}$ & $\begin{array}{l}\text { Pollinisateurs et disperseurs de graines } \\
\text { (Gilbert, 1980; Terborgh, 1986). }\end{array}$ \\
\hline $\begin{array}{l}\text { Modificateurs du } \\
\text { milieu. }\end{array}$ & $\begin{array}{l}\text { Créent des structures ou des paysages } \\
\text { qui permettent l'installation } \\
\text { et le maintien d'autres espèces. }\end{array}$ & Gros herbivores (Owen - Smith, 1987). \\
\hline
\end{tabular}

Tableau 1 - Les principaux types d'especes clé de voúte et leurs modes d'action.

la coexistence d'espèces potentiellement compétitives. Ce constat, indépendamment de toute théorisation autour du concept "d'espèce-clé", est d'importance pour la biologie de la conservation ou l'écologie de la restauration : la gestion des faunes et flores privées de leurs prédateurs "habituels" demande une intervention humaine. Le mythe de la Nature qui se régule d'ellemême a vécu. Il aura fallu plus de vingt ans pour que les professionnels de la protection de la Nature intègrent peu à peu cette leçon... quant aux amateurs, fascinés par telle ou telle espèce-phare, il leur reste encore du chemin à faire !
Après les prédateurs comme espèces clés, il était logique de rechercher de telles espèces parmi les étages trophiques inférieurs, et spécialement parmi les plantes, producteurs primaires et ressources de base pour les chaînes trophiques. C'est ce qu'a fait Terborgh (1986) avec succès, même si les tentatives de généralisation pratique du concept de ressources-clé peuvent être contestées (Gautier-Hion et Michaloud, $\left.1989^{2}\right)$. est bien démontré que certaines de ces res. sources-clé dépendent pour leur renouvellement ou leur expansion d'autres espèces
Dans le même esprit, dès lors qu'il associées - pollinisateurs, disperseurs de graines -, c'est-à-dire du mutualisme ${ }^{3}$, on va découvrir des espèces clés de voûte à travers cette fonction (Gilbert, 1980 ; Terborgh, 1986). En fait, parce que le mutualisme implique une association, une interdépendance, on peut se demander si, dans ce cas, le concept ne devrait pas s'appliquer à l'association elle-même plutôt qu'à l'une ou l'autre espèce prise séparément, espèce-ressource ou mutualiste. Le même type de dérive a pu être proposé par Brown et Heske (1990) avec le concept de "guilde-clé", qui associe plusieurs espèces assurant la même fonction - dans le cas cité celle de "rongeur granivore".

Enfin, au-delà des rôles de mangeurs ou de mangés, certaines espèces ont une fonction structurante, donc d'importants effets sur beaucoup d'autres espèces, parce qu'elles affectent ou déterminent la structure du milieu. C'est le cas des arbres, des élé. phants, des castors. On parle alors de "modificateurs du milieu". De fait, les éléphants constituent pour les savanes africaines aussi bien des herbivores-clé que des "ingénieurs" ou plutôt des "agenceurs écosystémiques" ("ecosystem engineers" de Jones et al., 1994) : il n'est pas aussi facile que cela de dissocier la fonction trophique et la fonction mécanique ${ }^{5}$.

\section{LES DÉRIVES DE SENS ET RAPPELS À L'ORDRE}

Pour Mills, Soulé et Doak (1993) le terme d'espèce clé de voûte est appliqué de façon beaucoup trop large, trop vague pour être de quelque utilité en biologie de la conservation ou en biologie de la restauration. Ils vont d'ailleurs plus loin : ils tiennent pour dangereux le recours à un cadre de référence flou pour l'élaboration de stratégies de conservation (ou de restauration puis-je ajouter ici).

De fait, parce qu'il y a plusieurs définitions pour les espèces clés, parce que différents auteurs l'utilisent dans un esprit différent, il y a matière à confusion. 
Ainsi, dans la définition de Robert Paine, les choses sont bien claires : il s'agit de prédateurs qui coiffent un réseau trophique et favorisent la coexistence d'espèces potentiellement compétitives parce qu'ils exercent une prédation préférentielle sur une espèce compétitivement dominante. Donc le retrait de tels prédateurs entraîne généralement une chute de diversité du fait de l'exclusion compétitive de plusieurs espèces.

Avec la loutre de mer et les oursins de Duggins, (1980) et Estes, Smith et Palmisano, (1978) les choses sont un peu différentes. En l'absence de loutres les oursins prolifèrent et, par leur action de broutage, changent le paysage végétal sous-marin et la faune associée. Le relais de l'action sur le peuplement benthique est un surpâturage contrôlé ou non.

Avec les fourmis de feu Solenopsis germinata de Risch et Carrol (1982) on s'écarte encore du sens originel : il s'agit d'un prédateur non spécialisé qui s'en prend à des herbivores faiblement compétitifs. Son absence entraîne non pas une régression de certaines populations et la disparition d'espèces mais, au contraire, l'accroissement des effectifs et de la richesse spécifique au sein du peuplement d'arthropodes défavorables aux cultures.

Dans tous ces cas le point de départ est empirique ou expérimental. Ce fut très clairement l'origine du concept dans les travaux et la réflexion de Robert Paine.

Avec les travaux de R.D. Holt $(1977,1984)$ la perspective est clairement théorique et l'approche celle de la modélisation mathématique. Il montre que, lorsque la proie préférée est capable de maintenir son abondance, la structure du peuplement est affectée dans un sens différent de celui observé par Bob Paine : le prédateur, ainsi entretenu à un niveau de densité élevé, peut réduire ou exclure d'autres populations de proies. Ici, l'espèce-clé n'est plus le prédateur mais la proie "prédateurs-tolérante". Comme dans le cas des fourmis de feu, l'éradication de l'espèce-clé se traduit par un accroissement de la diversité spécifique du peuplement associé. Théorique au départ cette situation a été observée en zone arctique à la suite de l'introduction d'une espèce de lapin qui, après avoir favorisé l'accroissement de la population de lynx, a entraîné l'effondrement d'autres espèces-proies de ce carnivore.

Et puis, on l'a vu, les espèces-clés ne se limitent pas aux seuls prédateurs. Ainsi, on a pu en découvrir chez des proies - on parle de "ressources-clé" (Terborgh, 1986 ; Gilbert, 1980), chez des mutualistes (voir Gilbert, 1980, qui parle de "mobile links" à propos d'animaux qui favorisent la persistance de plusieurs espèces de plantes, lesquelles favorisent à leur tour celles d'autres animaux et réseaux alimentaires entiers) et même chez des espèces qui agissent, non pas directement par le jeu de leurs relations trophiques, mais indirectement en affectant le milieu lui-même, les "agenceurs écologiques" de Jones, Lawton et Shachak (1994) (encadré).

Peut-on d'ailleurs raisonnablement imaginer des changements sensibles dans la

\section{LES INGÉNIEURS OU AGENCEURS ÉCOLOGIQUES}

Ce sont "des organismes qui modulent directement ou indirectement la disponibilité des ressources (autres qu'eux-mêmes) d'autres espèces en transformant le milieu".

L'archétype de ces ingénieurs écologiques c'est... non, pas I'homme : le castor ! Mais pourquoi pas I'homme au fait? On y reviendra dans le texte.

Développant l'idée, Jones et al. (1994) en viennent à distinguer différents types d'agenceurs écologiques, et notamment des agenceurs autogéniques et des agenceurs allogéniques. Les premiers agissent par le biais de leur propre transformation d'un état à un autre (développement d'une forêt qui modifie le microclimat et le flux des ressources pour d'autres espèces: l'édification d'un récif corallien) tandis que les seconds le font activement en transformant le milieu extérieur (alligators créant des mares favorables à d'autres espèces; lapins ou blaireaux creusant des terriers profitables à bien d'autres animaux; castors ... cf. figure 3 ).
2. Terborgh a fait des figuiers des ressources-cles pour de nombreux vertébrès frugivores des foréts tropicales: parce que certaines espèces de figuiers fructifient toute l'année elles permettent à ces dernières de passer la saison sèche. Gautier-Hion et Michaloud (1983) ont contesté la valeur générale de cette affirmation : ressources-clés à Panama, les figuiers ne le sont nullement dans les grandes forêts tropicales d'Afrique de l'ouest.

3. On appelle mutualisme toute relation entre deux organismes d'especes différentes qui se traduit par des effets positifs pour l'un et l'autre. Par exemple, il $y$ a mutualisme entre le bourdon et les fleurs qu'il pollinise : ce faisant, le bourdon recueille du nectar dont il se nourrit tandis que la fleur bénéficie de la pollinisation assurée par le transport des grains de pollen d'une fleur à l'autre.

4. Une guilde est un ensemble d'espèces apparentées (par exemple des rongeurs comme dans le cas cité ; des oiseaux, etc.) qui exploitent, dans un mème écosystème. le méme type de ressources alimentaires (graines, insectes...).

5. Cette dernière fonction peut ètre tres importante. Ainsi, pour Owen-Smith (1987), l'extinction de près de la moitiè des genres de mammifères d'une masse corporelle comprise entre 5 et $1000 \mathrm{~kg}$ à la fin du pléistocène aurait résulté de l'élimination des grands herbivores (plus de $1000 \mathrm{~kg}$ ). Ceux-ci, en effet, contribuaient à maintenir des paysages de savane herbeuse ouverts. Leur disparition aurait entrainé le boisement, voire une reforestation de vastes espaces, devenus ainsi hostiles à beaucoup d'especes mammaliennes. 
dynamique d'un système écologique - tels que la disparition ou le rétablissement d'une espèce-clé - qui n'impliquent pas presque nécessairement des effets indirects sur le milieu?

Considérons par exemple les expériences rapportées par Brown et Heske (1990) à propos de la guilde-clé constituée par trois espèces de Dipodomys ou rat-kangourou. Nous sommes dans le désert de Chihuahua, au sud-est de l'Arizona : le pay. sage est caractérisé par une végétation arbustive. Dans les parcelles expérimentales d'où l'on a exclu les rats-kangourou on observe un changement profond de la végétation, avec la mise en place progressive et durable d'une formation herbeuse. Celle-ci est dominée par une espèce pérenne, Eragrostris leh. mannia, dont l'abondance a été multipliée par vingt, et une annuelle, Aristida adscensionis, qui a triplé. Ces parcelles expérimentales sont colonisées par des rongeurs granivores, du genre Reithrodontomys caractéristiques des milieux herbacés. L'analyse des déterminismes en cause dans les changements survenus après l'élimination des rats-kangourou fait ressortir deux types de processus, l'un d'ordre biotique - la prédation exercée sur les graines - et l'autre d'ordre physique - l'impact sur la structure du sol. C'est ce dernier type d'action qui jouerait le rôle majeur sur le long terme (dizaine d'années).

L'effet biotique immédiat s'explique par la sélection qu'effectuent les rats-kangourou vis-à-vis des graines de grande taille. La suppression de ces derniers favorise les plantes annuelles à grandes graines, qui croissent en nombre et entraînent par compétition la régression des espèces annuelles à petites graines. Celles-ci profitaient en effet de la prédation sélective exercée par les rats-kangourou sur des espèces compétitivement dominantes. La disponibilité accrue des grandes graines, comme l'effacement de la compétition émanant des rats-kangourou, permettent de rendre compte, au moins en partie, du succès des Reithrodontomys.
Mais ce sont les effets physiques qui, à l'échelle de la dizaine d'années, paraissent jouer le rôle majeur dans la transformation du paysage végétal. Les rats-kangourou déplacent en effet une quantité importante de terre au cours de leurs activités de creusement de terriers et d'enfouissement des graines. Ces remaniements doivent faciliter la décomposition de la litière et l'établissement de nombreuses plantes annuelles. À l'inverse, la disparition ou la réduction de ces effets entraînées par l'élimination des rats-kangourou ont favorisé le développement de hautes herbes puis la colonisation par des espèces de rongeurs caractéristiques de milieux herbacés.

Ainsi, effets biotiques (prédation/ compétition) et effets physiques (altération du sol puis de la couverture végétale) ne sont pas aisément dissociables.

I- Les ingénieurs autogéniques

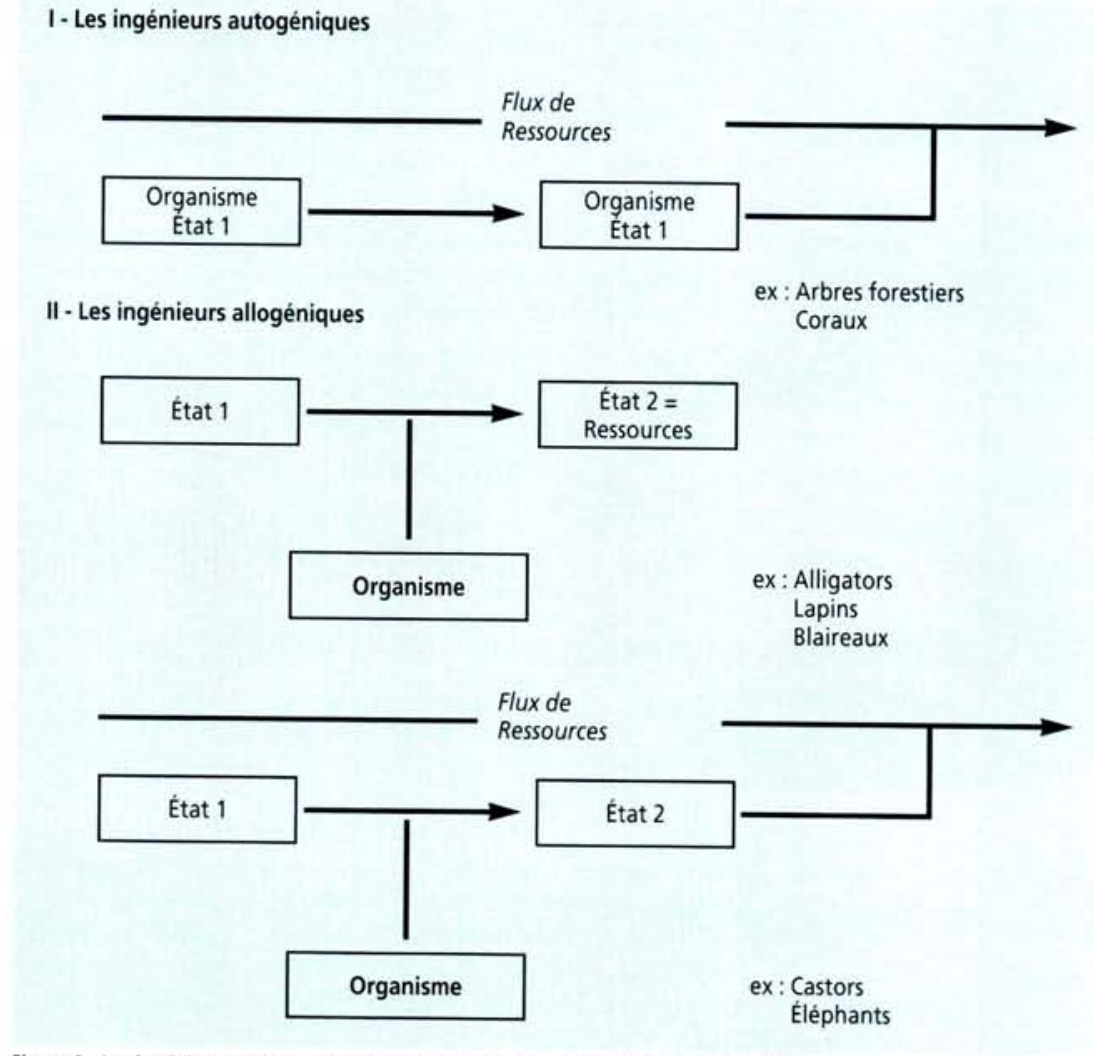

II- Les ingénieurs allogéniques Coraux

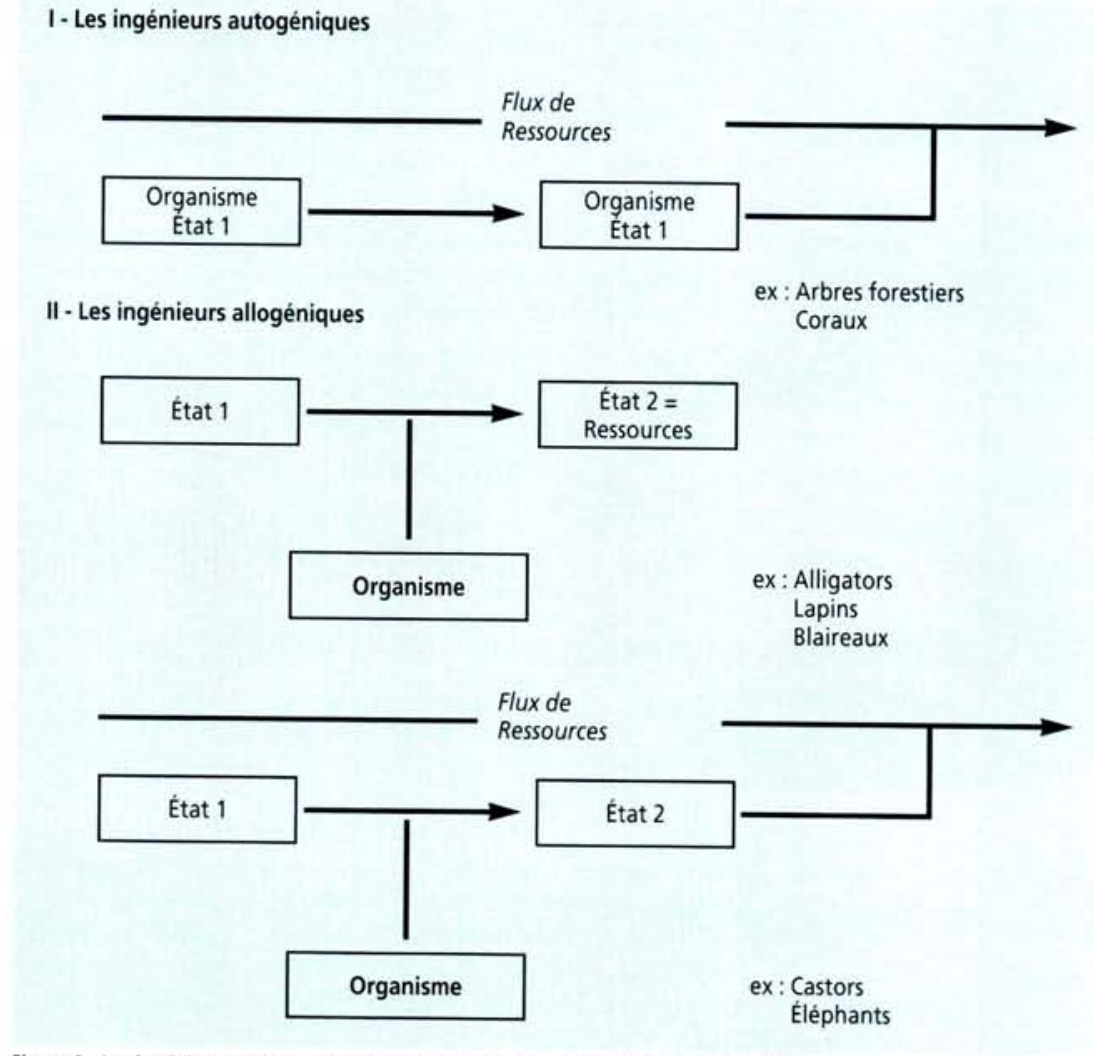

ex: Alligators Lapins Blaireaux

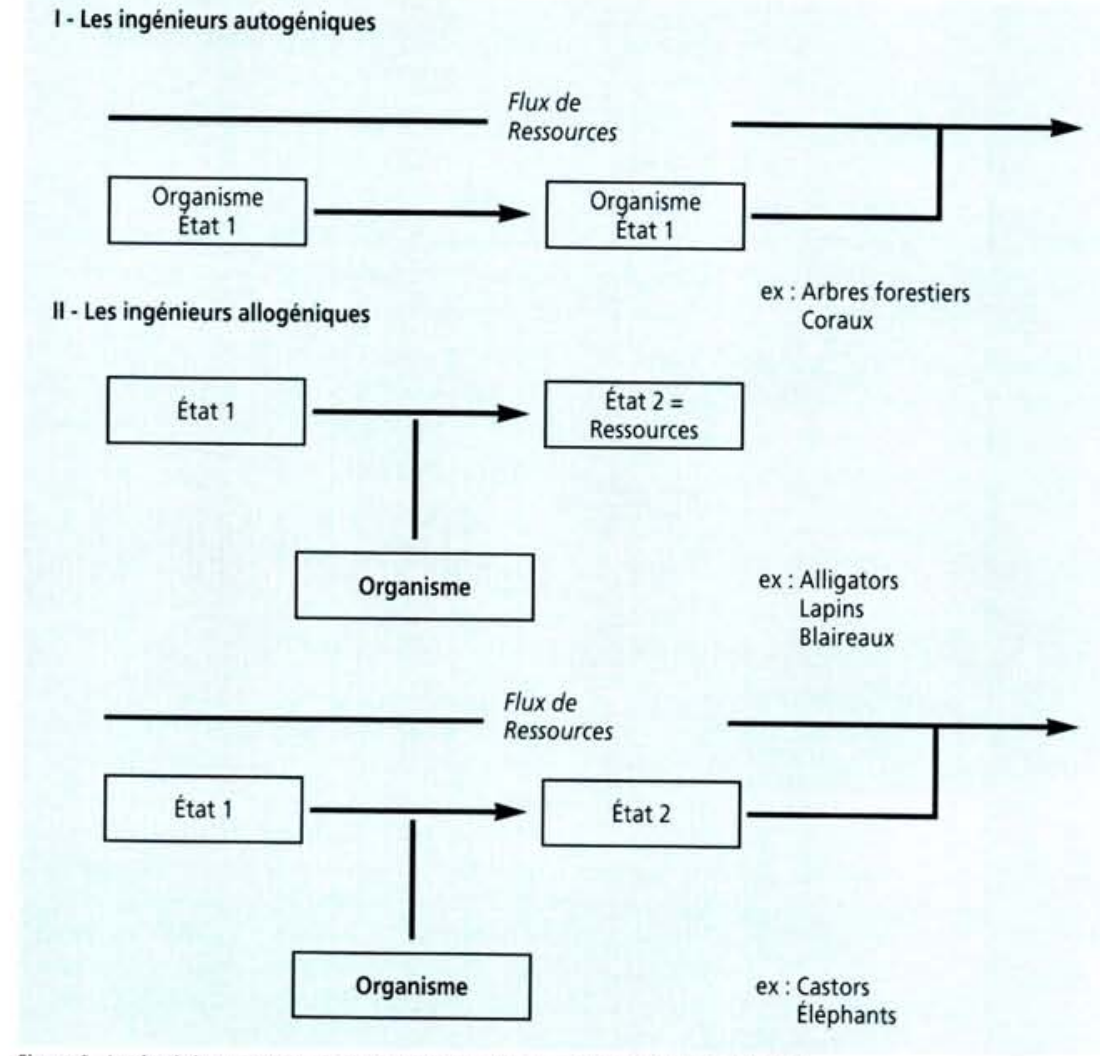

Figure 3 - Les ingénieurs ou agenceurs écologiques de Jones, Lawton et Shachak (1994).

\section{CLÉ ... OU IMPASSE ? D'UNE THÉORIE ABUSIVE \\ À LA PRATIQUE : LES LEÇONS À TIRER}

Avant que le concept d'espèce-clé ne devienne une pièce centrale dans la protection de la biodiversité ou la restauration des milieux il faudrait, comme le soulignent Mills et al. (1983), que l'on soit capable de le définir clairement.

On comprend la fascination première pour une recette miracle : l'espèce-clé, quel rêve ! L'essentiel des efforts de recherche et des ressources en matière de conservation ou de restauration porte sur un petit nombre d'espèces menacées d'extinction : que celles-ci soient des espèces-clés et voilà un redressement de situation inespéré. Sinon il faudra bien parler de l'arbitraire 
des choix... et de la faible efficacité globale d'une telle stratégie.

Mais on l'a vu, la définition des espèces-clés n'est pas une chose commode, aussi objective qu'on pouvait l'espérer. Pire : la même espèce reconnue comme clé ici, ne le sera pas là (voir Gautier-Hion et Michaloud, 1989 et note 1). C'est donc une illusion dangereuse. Il est dangereux en effet de généraliser trop vite, à partir d'informations fragmentaires ou biaisées : c'est oublier l'histoire des systèmes écologiques et donc leur spécificité. Et quant on songe d'autre part au contexte social et humain - c'est I'homme qui restaure, pour lui-même - cela s'aggrave encore.

Quelles leçons pouvons-nous tirer de ces rapides considérations sur les limites de la théorie des espèces-clés ?

Tout d'abord il convient d'insister, paradoxalement peut-être, sur les vertus de la théorie. Le constat de difficultés dans la mise en cuvre directe de ses principes voire d'impossibilité à le faire n'équivaut pas à un jugement d'inutilité. La théorie est nécessaire par le cadre structurant qu'elle apporte : elle aide à formuler les questions ; elle permet de déceler les risques d'erreur ou d'égarement ; elle est formatrice. Mais cela veut dire aussi qu'une fois assimilé, le cadre théo- rique peut être oublié : il a joué son rôle de guide ou de garde-fou et l'on est en mesure de s'engager dans l'action sans préoccupation particulière de conformisme orthodoxe. Bref, le bricolage du génie écologique, des écotechniques (qui suppose un bagage théorique de haut niveau) n'est pas plus à rejeter que celui de l'Évolution, si bien réhabilité par François Jacob (1977)!

En ce qui concerne la conservation ou la restauration de la biodiversité ou d'espèces favorisant le développement de peuplements particuliers on peut tirer trois types de leçons utiles.

La première, qui prend acte de l'idée de système, d'interactions entre espèces, de complexité, conduit à revenir à l'écologie des peuplements (Barbault, 1992, Barbault et Hochberg, 1992). De fait, comme le soulignait Western (1982), " How can we safeguard biodiversity unless we understand how communities are assembled and maintained? ". À partir de réseaux d'espèces en interaction on peut faire des hypothèses sur les risques d'extinction en cascade (figure 4, d'après Pimm, 1991) et, inversement, dans des projets de restauration ciblant sur telle ou telle espèce, on pourra en déduire les contraintes à respecter (restaurer les popu-

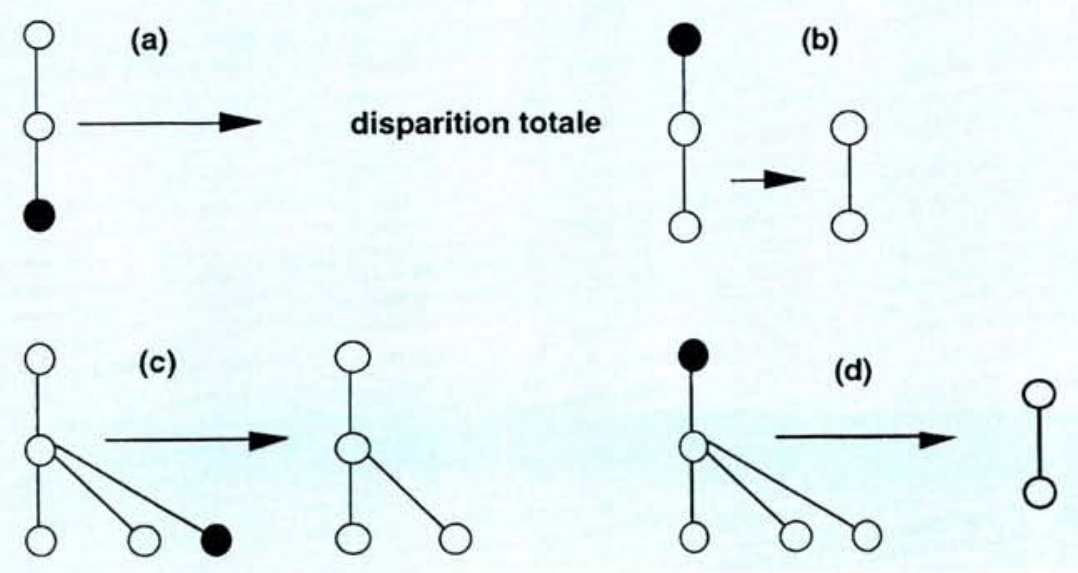

Figure 4 - Les effets de l'élimination d'une espèce sur d'autres dépendent de la structure du réseau trophique et de la position de celle-ci dans le réseau. L'extinction d'une espèce de plante à la base d'une chaine trophique simple peut entrainer la disparition totale (a), tandis que celle d'un prédateur de sommet peut ne pas avoir d'effet (b). Si le réseau trophique est plus complexe en revanche la perte d'une espèce de plante peut rester sans conséquences (c) alors que celle d'un prédateur (d) peut entrainer indirectement l'extinction de plusieurs espèces de plantes (d'après Pimm, 1992). 
lations d'espèces qui conditionnent la présence de celle que l'on souhaite).

Dans le même esprit, et plus généralement, il faut insister sur l'importance du contexte géographique et climatique et sur le rôle de l'hétérogénéité spatio-temporelle. Revenons à l'exemple du système "rongeurs/graines/désert arbustif" manipulé par Brown et Heske (1990). Les parcelles expérimentales étaient situées à proximité de la zone de transition entre le désert et la prairie de sorte que la simple suppression d'espèces, appelées "clés" pour cela, a pu aisément faire basculer le système d'un type de végétation à l'autre. Il n'est pas évident, soulignent les auteurs, que l'on eut obtenu des résultats aussi marquants en pratiquant la même expérimentation au cœur du Chihuahua, loin de conditions permettant facilement le glissement vers une végétation herbacée.

Ainsi, l'écologue de la restauration sera nécessairement aussi un écologue du paysage. De fait, le cadre spatial est une dimension-clé des problèmes qui lui sont posés. Déjà évident quand on s'en tient à leur approche écologique cela devient central, incontournable, quand on entend légitimement prendre en compte leur dimension sociale et économique pour une restauration durable, c'est-à-dire acceptée par les populations humaines concernées.

La seconde était déjà formulée clairement et vigoureusement par les maitres, MacArthur (1972) et Paine $(1980,1992)$ : plutôt que de s'aveugler sur la recherche d'espèces plus importantes que d'autres il vaudrait mieux distinguer et repérer les interactions importantes, c'est-à-dire les interactions fortes. Paine (1992) souligne que dans les réseaux trophiques il y a généralement peu d'interactions fortes ${ }^{6}$, c'est-àdire peu d'espèces à connections étroites - la plupart n'étant que faiblement interactives. Retenons le ciblage déplacé de l'espèce à l'interaction ${ }^{7}$ !

La troisième leçon émerge de l'idée de fonction (voir Walker, 1992) et d'espèces substituables. Elle est complémentaire de la précédente et, comme elle, elle tend à désacraliser les espèces : il y a d'abord des fonctions clés, que des espèces différentes peuvent remplir.

C'est une autre façon d'échapper au dilemme évoqué en introduction : prendre en compte l'ensemble des espèces qui constituent l'écosystème, au risque de se perdre dans une accumulation infinie de monographies d'espèces, ou bien réduire cet assemblage complexe à quelques compartiments fonctionnels, les niveaux trophiques. La première solution envisagée, celle dont traite le présent article est de concentrer les efforts de recherche sur quelques espèces particulièrement importantes, les espèces clé de voûte. La seconde solution qui se dégage maintenant à la discussion est d'affiner l'approche fonctionnelle (encadré) en subdivisant les niveaux trophiques en sousensembles plus précis, les guildes ou groupes fonctionnels. Cette approche conserverait l'orientation populationnelle prônée dans la première solution pour renouveler la perspective énergétique propre à l'étude des réseaux trophiques, trop "désincarnée" à notre goût dans les travaux classiques de flux d'énergie et de matière.

Le travail de Brown et Heske (1990) est une magnifique illustration du progrès conceptuel que cela représente : ne parlentils pas de guilde-clé en place d'espèce-clé ? C'est évidemment plus réaliste quand on sait la richesse spécifique des écosystèmes naturels : la plupart des fonctions écologiques y sont assumées généralement par plusieurs espèces, comme dans le cas des trois Dipodomys étudié par Brown et Heske. Cela veut dire, dans une perspective "écologie de la restauration", que ces trois espèces sont équivalentes en première approximation, et que l'une d'entre elles pourra restaurer l'écosystème désertique en ce qui concerne la fonction "mangeur de grosses graines et brasseur de sol". Cette idée de redondance écologique, c'est-à-dire d'espèces substituables, s'est peu développée en écologie, peut-être parce qu'elle heurtait le théorème classique, longtemps dominant, "une espèce = une niche". Il est pourtant intéressant de souligner ici que la théorie de la niche écologique est, par essence, une tentative de syn-

\section{L'IDÉE DE GROUPE FONCTIONNEL}

L'idée de groupes fonctionnels est apparue à plusieurs reprises dans l'histoire de la biologie puis de l'écologie. Elle s'est imposée aux écologues intéressés par la dynamique des communautés et la compréhension de ses déterminismes à la suite des renouvellements théoriques apportés par MacArthur et Wilson à la fin des années soixante : Ils parlent alors de guildes, assemblages d'espèces apparentées exploitant localement un même type de ressources (par ex. : oiseaux nectarivores). Elle a émergé également, sous une autre forme, dans les travaux de théoriciens intéressés par la structure et la complexité des réseaux trophiques: les espèces occupant à première vue la même position dans le réseau ( $c^{\prime}$ est-à-dire mangeant les mêmes proies et étant victimes des mêmes prédateurs) sont regroupées sous le vocable $\mathrm{d}^{\prime}$ " espèces trophiques " (Briand et Cohen, 1987). Cependant, indépendamment de tout cela et bien antérieurement d'autres biologistes, naturalistes ou physiologistes, avaient développé le concept de type fonctionnel et défini, par exemple, des types fonctionnels chez les plantes. De fait, depuis les travaux de
Raunkiaer (1934), le concept de forme de vie s'est avéré très utile pour analyser l'action du climat sur la composition et la dynamique de la végétation (Box, 1981). Parce qu'elle repose sur des critères morphologiques facilement accessibles cette approche a été très appréciée. Cependant, parce que d'importants aspects de la végétation ne sont pas détectables par référence à la seule morphologie des plantes, il est vite apparu nécessaire d'y adjoindre d'autres caractères, reliés de manière prévisible à l'habitat et l'écologie des espèces (voir Grime, 1979).

Toutes ces approches sont intéressantes et devraient, au même titre que la réflexion sur les espèces clés de voûte, contribuer largement au progrès de l'écologie de la restauration. L'un de leurs apports majeurs est certainement, pour ce qui nous concerne ici, le concept de redondance fonctionnelle : quelles espèces sont fonctionnellement équivalentes? Dans quelle mesure et comment la structure et le fonctionnement d'un écosystème sont-ils affectés par la disparition de telle ou telle espèce ? 
thèse entre l'approche "populationnelle" et l'approche "fonctionnelle" : dès Elton (1927) la niche écologique était définie comme la place et la fonction de l'espèce dans le système considéré. Je vois là l'une ${ }^{8}$ des sources historiques de l'idée de groupe fonctionnel telle qu'elle est aujourd'hui préconisée pour aborder la dynamique des écosystèmes d'une manière à la fois opérationnelle et conforme aux acquis de la biologie des populations.

\section{CONCLUSION}

"Les conservationnistes et les gestionnaires de réserves naturelles ne devraient-ils pas accorder plus d'attention au rôle des agenceurs écologiques dans le maintien de l'intégrité des écosystèmes ? " écrivent Jones et al. (1984).

Certes ! Mais n'est-il pas étonnant - et révélateur - que l'on fasse du castor le paradigme de "l'agenceur écologique" plutôt que l'homme, espèce-clé par excellence dans le sens élargi que nous avons indiqué plus haut. Notre espèce n'apparaît ni dans la typologie des espèces clés de voûte de Bond (1993) ou de Mills et al. (1993), ni dans la réflexion de Jones et al. (1994) sur les “ingénieurs ou agenceurs d'écosystèmes".

Quand on réfléchit sur les apports de l'écologie théorique aux pratiques de restauration, quand on déplore les dégradations passées et à venir que l'homme impose aux écosystèmes, ce constat est instructif et milite en faveur de l'instauration d'une nouvelle fonction (ou d'une restauration, d'une réhabilitation de cette fonction)... celle d'ingénieur écologue !

En fait, l'une des faiblesses majeures de la fascination que l'on pourrait avoir pour une entrée à l'écologie de la restauration par les espèces clés est liée au cadre conceptuel dans lequel elles ont été conçues - et la représentation graphique des réseaux trophiques le montre bien : nous sommes dans un univers privé de toute structuration spatiale explicite et hors du temps, privé d'histoire (Wiens, 1989 ; Barbault, 1992 ; Barbault et Hochberg, 1992). Or la restau- ration des systèmes écologiques s'inscrit nécessairement dans un contexte spatial et temporel : l'histoire du système et sa géographie ont un poids décisif dans son fonctionnement, sa structure, sa dynamique. Il ne saurait y avoir de restauration efficace hors d'une réflexion nourrie de tout cela. D'ailleurs, cette remarque a une portée plus générale et vaut pour l'écologie théorique aussi bien que pour l'écologie de la restauration : une approche "écologie des paysages" "'impose (Wiens et al., 1993 ; Barbault, 1995).

\section{Références}

Barbault R. (1992). Ecologie des peuplements. Structure, fonctionnement et evolution, Paris, Masson.

Barbault R. (1994). Des baleines, des bactéries et des hommes, Paris, Odile Jacob.

Barbault R. (1995). Biodiversity dynamics : from population and community ecology approaches to a landscape ecology point of view, Landscape and Urban Planning, 31, 89-98.

Barbault R. et Hochberg M.E. (1992). Population and community level approaches to studying biodiversity in international research programs, Acta Oecologica, 13, 137-146.

Bond W.Y. (1993). Keystone species, pp 237-253, In E.D. Schulze et H.A. Mooney (Eds), Biodiversity and Ecosystem Function, Berlin, Springer-Verlag.

Box E.O. (1981). Macroclimate and plant forms : an introduction to predictive modelling in phytogeography, The Hague, Junk.

Briand F. et Cohen J.E. (1987). Environmental correlates of food chain length, Science, 238, 956-960.

Brown Y.H. et Heske E.Y. (1990). Control of a desert-grassland transition by a keystone rodent guild, Science, 250, 1705 1707.

Duggins D.O. (1980). Kelp beds and sea otters : an experimental approach, Ecology, 61, 447-453.

Elton C.S. (1927). Animal ecology, London, Sidgwick et Jackson. Estes J.A., Smith N.S. et Palmisano J.F. (1978). Sea otter predation and community organization in the western Aleutian islands, Alaska, Ecology, 59, 822-833.

Forman R.T.T. et Godron M. (1986). Landscape ecology, New York, Wiley.

Gautier-Hion A. et Michaloud G. (1989). Are figs always keystone resources for tropical frugivorous vertebrates? A test in Gabon, Ecology, 70, 1826-1833.

Gilbert L.E. (1980). Food web organization and the conservation of neotropical diversity, pp. 11-33 In M.E. Soulé et B.A. Wilcox (Eds), Conservation biology : an evolutionary-ecological perspective, Sunderland, MA, Sinauer.

Grime J.P. (1979). Plant strategies and vegetation process, Chichester, Wiley.

Holt R.D. (1977). Predation, apparent competition, and the structure of prey communities, Theor. Popul. Biol., 12, 197-229. Holt R.D. (1984). Spatial heterogeneity, indirect interactions, and the coexistence of prey species, Am. Nat., 124, 377-406. Jacob F. (1977). Evolution and Tinkering, Science, 196, 11611166.

Jones C.G., Lawton J.H. et Schachak M. (1994). Organisms as ecosystem engineers, Oikos, 69, 373-386.

Lefeuvre J.C. et Barnaud G. (1988). L'écologie du paysage :
6. Mac Arthur (1972) parle en fait de " strong interactor "à propos d'espéces dont la suppression pourrait avoir des effets dramatiques. C'est toujours l'idée d'espèce-clè, mais définie plus largement.

7. « Emphasizing strengths of interactions instead of a keystone/nonkeystone dualism is more than a semantic improvement; it recognizes the complexity, as well as the temporal and spatial variability, of interactions n (Mills et al. 1993).

8. L'autre source étant l'idée de type fonctionnel telle qu'elle fut pratiquée par divers botanistes ou écophysiologistes (encadré 4).

9. L'écologie du paysage est une branche de l'écologie relativement récente, qui possède sa société internationale, ses revues spécialisées (Landscape Ecology: Landscape and Urban Planning), ses traités (Naveh et Lieberman, 1984 ; Forman et Godron, 1986, etc.). Elle a pour but l'étude des structures et des processus propres aux milieux hètérogènes occupant une échelle géographique étendue (Lefeuvre et Barnaud, 1988 ; Turner, 1989). C'est une discipline de base pour l'écologie de la restauration. 
mythe ou réalité ? Bull. Écologie, 19, 493-522.

Mac Arthur R.H. (1972). Geographical Ecology : patterns in the distribution of species, Harper et Row.

Mills L.S., Soulé M.E. et Doak D.F. (1993). The keystone - species concept in ecology and conservation, Bioscience, 43, 219. 224.

Naveh Z. et Lieberman A.S. (1984). Landscape ecology. Theory and applications, New York, Springer-Verlag.

Owen-Smith N. (1987). Pleistocene extinctions: the pivotal role of megaherbivorous, Paleobiology, 13, 351-362.

Paine R.T. (1966). Food web complexity and species diversity, Am. Nat, 100, 65-75.

Paine R.T. (1969). A note on trophic complexity and community stability, Am. Nat., 103, 91-93.

Paine R.T. (1980). Food webs : linkage, interaction strength and community infrastructure, J. Anim. Ecol., 49, 667-685.

Paine R.T. (1992). Food-web analysis through field measurement of per capita interaction strength, Nature, 355, 73-75.

Pimm S.L. (1991). The Balance of Nature ? Ecological issues in the conservation of species and communities, Chicago, The University of Chicago Press.
Raunkiaer C. (1934). The life form of plants and statistical plant geography, Oxford, Clarendon.

Risch S.J. et Carroll C.R. (1982). Effects of a keystone predaceous ant, Solenopsis germinata, on arthropods in a tropical agroecosystem, Ecology, 63, 1979-1983

Terborgh J. (1986). Keystone plant resources in the tropical forest, pp 320-340, In M.E. Soulé (Ed.) Conservation biology the science of scarcity and diversity, Sunderland, MA, Sinauer.

Turner M.G. (1989). Landscape ecology : the effect of pattern on process, Annu. Rev. Ecol. Syst., 20, 171-197.

Walker B.H. (1992). Biodiversity and Ecological Redundancy, Conservation Biology, 6, 18-23.

Western D. (1992). The biodiversity crisis : a challenge for biodiversity, Oikos, 63, 29-38.

Wiens J.A. (1989). The ecology of bird communities, Cambridge, Cambridge Univesity Press.

Wiens J.A., Stenseth N.C., Van Horne B. and Ims R.A. (1993) Ecological mechanisms and landscape ecology, Oikos, 66, 369 . 380.

\section{ABSTRACT : The Concept of Keystone Species in Restoration Ecology : key... or impasse ?}

Whether we seek to understand how they function, or whether we intend to manage or restore them, ecological systems confound us with the multitude of species by which they are composed - this is a major problem. Trying to surmount this obstacle is frustrating because the options to which we can turn such as the reduction of these incredibly diverse assemblages into a few trophic levels or the indefinite accumulation of species monographs are equally unsatisfying alternatives.

Born in the 1960's, the concept of keystone species is once again attracting interest: among the current debates concerning the conservation of biodiversity, it appears to offer a promising route that can access and deal with the complexity described above.

It postulates that certain species are more important than others because they are situated at intersections within the trophic network and there play a pivotal role. Thus the concept leads us to believe that we can focus our attention and efforts on a small number of species, allowing us to neglect all the other species that are directly or indirectly dependent upon them.

Unfortunately, it appears that this concept leaves more problems unresolved than it can solve. However, even if it does not represent in itself a miracle tool, directly applicable to restoration ecology, it reinforces the underlying assumptions upon which it is built :

- the idea of an ecological system (as opposed to the first perception that a multitude of species exist, unconnected) :

- the idea of interactions between species (a logical consequence of the first point) ;

- the concept of a population-environment system that integrates all the knowledge of population biology.

Perhaps it is not a miracle key, but far from leading us into an impass, the concept of keystone species compels us to explore and consider interesting possibilities in the field of restoration ecology. 